\title{
Squeezing Flow of Micropolar Nanofluid between Parallel Disks
}

\author{
Sheikh Irfanullah Khan ${ }^{1}$, Syed Tauseef Mohyud-Din ${ }^{1 *}$, and Yang Xiao-Jun ${ }^{2}$ \\ ${ }^{l}$ Faculty of Sciences, HITEC University, Taxila Cantt, Pakistan \\ ${ }^{2}$ School of Mechanics and Civil Engineering, China University of Mining and Technology, Xuzhou 221116, China
}

(Received 28 April 2016, Received in final form 23 June 2016, Accepted 23 June 2016)

\begin{abstract}
In the present study, squeezing flow of micropolar nanofluid between parallel infinite disks in the presence of magnetic field perpendicular to plane of the disks is taken into account. The constitutive equations that govern the flow configuration are converted into nonlinear ordinary differential with the help of suitable similarity transforms. HAM package BVPh2.0 has been employed to solve the nonlinear system of ordinary differential equations. Effects of different emerging parameters like micropolar parameter $K$, squeezed Reynolds number $R$, Hartmann number $M$, Brownian motion parameter $N b$, thermophoresis parameter $N t$, Lewis number $L e$ for dimensionless velocities, temperature distribution and concentration profile are also discussed graphically. In the presence of strong and weak interaction (i.e. $n=0$ and $n=0.5$ ), numerical values of skin friction coefficient, wall stress coefficient, local Nusselt number and local Sherwood number are presented in tabulated form. To check the validity and reliability of the developed algorithm BVPh2.0 a numerical investigation is also a part of this study.
\end{abstract}

Keywords : HAM BVPh2.0, micropolar nanofluid, squeezing flow, strong and weak interactions, skin friction coefficient

\section{Introduction}

Non-Newtonian fluids play a vital role in industry and engineering due to its diverse characteristics in nature. In comparison to Navier-Stokes equations, the governing equations of such fluids are higher in order and highly nonlinear [1-3]. Non-Newtonian fluids cannot be examined by virtue of a single expression. Due to this fact, rheological parameter involved in the constitutive equations of such fluids adds complexity in the arising systems. Regardless of all these complexities, several researchers [4-8] studied the flow of non-Newtonian fluids in different geometrical configurations. However, the microscopic properties like micro-rotation and rotation-inertia of micropolar fluids be different from the other non-Newtonian fluids. Eringen [9] was the first one who proposed the theory of micropolar fluids. Micropolar fluids are very important for the flow of liquid crystals, fluid with additives, colloidal suspension, animal blood and suspension solutions etc. In this regard, many researchers [10-19]

(C) The Korean Magnetics Society. All rights reserved.

*Corresponding author: Tel: +923235577701

Fax:+92514908154, e-mail: syedtauseefs@hotmail.com encountered the flow of micropolar fluids by taking into account various configurations.

In addition, squeezing flows are investigated frequently in polymer processing, hydrodynamic machines, lubrication process, compression and injection modelling etc. Ishizawa [20] has been discussed the unsteady flow between two parallel disks with arbitrary varying gap width and derived a perturbative solution for the flow caused by the disks moving towards each other. Squeezing flows of Newtonian liquid films has been investigated by Grimm [21]. He gave a numerical solution by taking fluid inertia effect into account and compared his results with those done by Ishizawa [20]. Wang [22] applied perturbation method to solve the resulting two point boundary value problem on squeezing flow of viscous fluid between elliptic plates. Flow induced by the squeezing motion of elliptic plates have been examined by Usha and Sridharan [23]. They derived exact solutions and found that the hydrodynamic forces acting on the wall surface are more slanted in wave form as the amplitude increases. Recently, Rashidi et al. [24] derived an analytic solution for squeezing axisymmetric flow of a viscous fluid between infinite parallel disks by using homotopy analysis method (HAM). 
Furthermore, in the last two decades a new class of fluid termed as "nanofluid" has much attention to the engineering researchers. These fluids are engineered colloidal suspensions of nanoparticles with sizes typically 1-100 nm dispersed in a base fluid such as water, toluene, ethylene glycol and oil. Choi [25], initially verified that with inclusion of these nanoparticles in a base fluid, enhances the thermal conductivity of the fluids. To highlight the significant effects of Brownian motion and thermophoretic diffusion of nanoparticles, a mathematical model was discussed by Buongiorno [26]. In Buongiorno's model, the thermal conductivity and the viscosity are taken to be constant. This model takes into consideration the Brownian motion and the thermophoresis effects which is different from the other models used. This model is called two phase model due the presence of Brownian motion and the thermophoresis, i.e. the temperature and concentration equations are coupled due to the presence of these two terms. Many researchers used the Buongiorno's model to analyze the flow of nanofluids in various geometries. Some relevant studies can be seen in [27-30] and the references therein.

According to best of author's knowledge that there is no study available in the literature regarding squeezing flow of micropolar nanofluid between parallel disks in the presence of applied magnetic field perpendicular to the plane of disks. An exact solution is unlikely; due to highly nonlinearity of these problems, so, many analytical techniques such as Homotopy perturbation method, Variational iteration method, variation of parameters method, etc., have been established to approximate the solutions. Liao presented a new form of HAM and implemented it on as a novel analysis to the field [31-33]. Different researchers used BVPh2.0 package to solve the equations of various types [34, 35]. Therefore, the above problem is solved by Homotopy Analysis Method (HAM) using the package BVPh2.0. A numerical solution using RK-4 method is also obtained. An excellent agreement found between the developed algorithm and numerical solutions.

\section{Mathematical Analysis}

The influence of magnetic field practiced vertically and proportional to $B_{0}(1-a t)^{1 / 2}$ is considered for two-dimensional axisymmetric flow of an incompressible nanofluid between parallel disks, where the magnetic field is negligible for low Reynolds numbers. $\tilde{C}_{w}$ and $\tilde{C}_{h}$ represents the nanoparticles concentration, while $\tilde{T}_{w}$ and $\tilde{T}_{h}$ are the constant temperatures at lower and upper disks respectively. It is important to mention that, water is taken as a base fluid. The effects of micropolar parameter and squeeze Reynolds number are considered for dimensionless velocities (radial velocity and angular velocity). The upper disk is moving with a velocity $\frac{a H}{2 \sqrt{1-a t}}$ towards or away from the lower disk as shown in Fig. 1.

Thus, the governing equations for two-dimensional unsteady flow and heat transfer of a viscous fluids are

$$
\begin{aligned}
& \frac{\partial \hat{u}}{\partial r}+\frac{\hat{u}}{r}+\frac{\partial \hat{w}}{\partial z}=0, \\
& \frac{\partial \hat{u}}{\partial t}+\hat{u} \frac{\partial \hat{u}}{\partial r}+\hat{w} \frac{\partial \hat{u}}{\partial z}=-\frac{1}{\rho} \frac{\partial \tilde{p}}{\partial r}+\frac{1}{\rho}(\mu+k) \\
& \left(\frac{\partial^{2} \hat{u}}{\partial r^{2}}+\frac{1}{r} \frac{\partial \hat{u}}{\partial r}-\frac{\hat{u}}{r^{2}}+\frac{\partial^{2} \hat{u}}{\partial z^{2}}\right)-\frac{k}{\rho} \frac{\partial \tilde{N}}{\partial z}-\frac{\sigma}{\rho} B^{2}(t) \hat{u},
\end{aligned}
$$

$$
\begin{array}{r}
\frac{\partial \hat{w}}{\partial t}+\hat{u} \frac{\partial \hat{w}}{\partial r}+\hat{w} \frac{\partial \hat{w}}{\partial z}=-\frac{1}{\rho} \frac{\partial \tilde{p}}{\partial z}+\frac{1}{\rho}(\mu+k) \\
\left(\frac{\partial^{2} \hat{w}}{\partial r^{2}}+\frac{1}{r} \frac{\partial \hat{w}}{\partial r}+\frac{\partial^{2} \hat{w}}{\partial z^{2}}\right)-\frac{k}{\rho}\left(\frac{\partial \breve{N}}{\partial r}+\frac{\breve{N}}{r}\right),
\end{array}
$$$$
\frac{\partial \breve{N}}{\partial t}+\hat{u} \frac{\partial \breve{N}}{\partial r}+\hat{w} \frac{\partial \breve{N}}{\partial z}=\frac{\gamma}{\rho j}\left(\frac{\partial^{2} \breve{N}}{\partial r^{2}}+\frac{1}{r} \frac{\partial \breve{N}}{\partial r}+\frac{\partial^{2} \breve{N}}{\partial z^{2}}-\frac{\breve{N}}{r^{2}}\right)
$$$$
-\frac{k}{\rho j}\left(2 \breve{N}+\frac{\partial w}{\partial r}-\frac{\partial u}{\partial z}\right)
$$

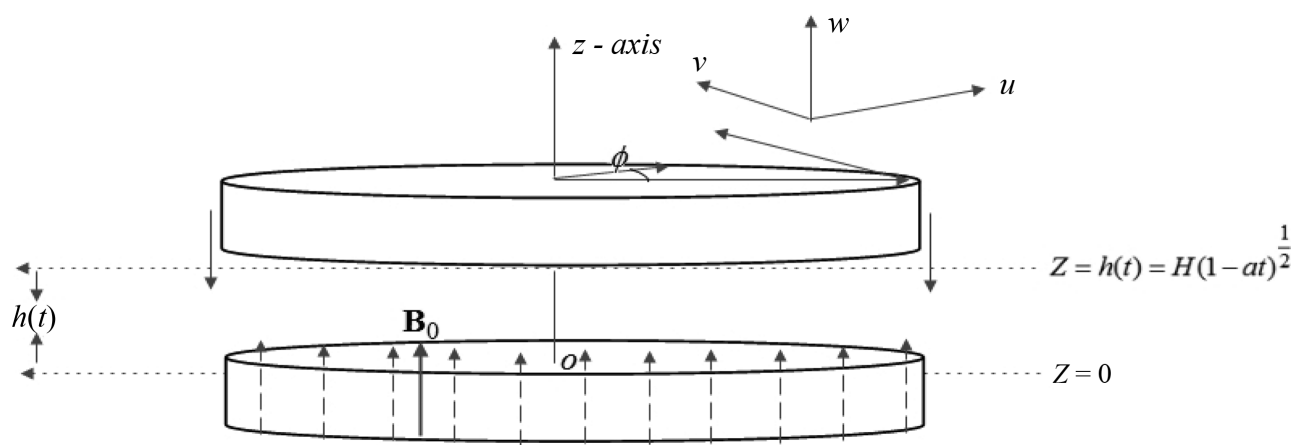

Fig. 1. Schematics diagram of the problem. 


$$
\begin{aligned}
& \frac{\partial \tilde{T}}{\partial t}+\hat{u} \frac{\partial \tilde{T}}{\partial r}+\hat{w} \frac{\partial \tilde{T}}{\partial z}=\alpha\left(\frac{\partial^{2} \tilde{T}}{\partial r^{2}}+\frac{1}{r} \frac{\partial \tilde{T}}{\partial r}+\frac{\partial^{2} \tilde{T}}{\partial z^{2}}\right) \\
& +\tau\left(D_{B}\left(\frac{\partial \tilde{C}}{\partial r} \frac{\partial \tilde{T}}{\partial r}+\frac{\partial \tilde{C}}{\partial z} \frac{\partial \tilde{T}}{\partial z}\right)+\frac{D_{T}}{T_{m}}\left[\left(\frac{\partial \tilde{T}}{\partial r}\right)^{2}+\left(\frac{\partial \tilde{T}}{\partial z}\right)^{2}\right]\right), \\
& \frac{\partial \tilde{C}}{\partial t}+\hat{u} \frac{\partial \tilde{C}}{\partial r}+\hat{w} \frac{\partial \tilde{C}}{\partial z}=D_{B}\left(\frac{\partial^{2} \tilde{C}}{\partial r^{2}}+\frac{1}{r} \frac{\partial \tilde{C}}{\partial r}+\frac{1}{r^{2}} \frac{\partial^{2} \tilde{C}}{\partial \theta^{2}}\right) \\
& +\frac{D_{T}}{T_{m}}\left(\frac{\partial^{2} \tilde{T}}{\partial r^{2}}+\frac{1}{r} \frac{\partial \tilde{T}}{\partial r}+\frac{1}{r^{2}} \frac{\partial^{2} \tilde{T}}{\partial \theta^{2}}\right) .
\end{aligned}
$$

Subject to the auxiliary conditions

$$
\begin{aligned}
& \left.\left(\hat{u}=0, \hat{w}=\frac{d h}{d t}, \breve{N}=-n \frac{\partial u}{\partial z}\right)\right|_{z=h(t)}, \\
& \left.\left(\hat{u}=0, \hat{w}=-\frac{w_{0}}{\sqrt{1-a t}}, \breve{N}=-n \frac{\partial u}{\partial z}\right)\right|_{z=0}, \\
& \left.\left(\tilde{T}=\tilde{T}_{h}, \tilde{C}=\tilde{C}_{h}\right)\right|_{z=h(t)}, \\
& \left.\left(\tilde{T}=\tilde{T}_{w}, D_{B} \frac{\partial \tilde{C}}{\partial z}+\frac{D_{T}}{T_{m}} \frac{\partial \tilde{T}}{\partial z}=0\right)\right|_{z=0} .
\end{aligned}
$$

Where $n$ is a constant and $\gamma=\left(\mu+\frac{k}{2}\right) j$ and $j=\frac{v(1-a t)}{\alpha}$ are the reference lengths [18]. $\hat{u}$ and $\hat{w}$ are the velocity components in $r$ - and $z$-directions respectively and $\breve{N}$ is the azimuthal component of micro-rotation. $\tilde{C}$ the nanoparticle concentration, $\alpha$ thermal diffusivity, $\rho$ the density, kinematic viscosity $v$, pressure $\hat{p}$ and specific heat $C_{p}$. Further, the coefficient of mass diffusivity $D$, Brownian motion coefficient $D_{B}$, thermophoretic diffusion coefficient $D_{T}$, and $K_{T}$ is the thermal-diffusion ratio. $\tilde{T}, \tilde{T}_{m}$ correspondingly are the temperature and mean fluid temperatures. Furthermore, $\tau$ is the dimensionless parameter which describes the ratio of effective heat capacity of nanoparticles to heat capacity of the fluid and $w_{0}$ is the suction/injection velocity. In the current study we take $n=0$ and $n=0.5$, which describes the phenomena of strong and weak interactions respectively.

To non-dimensionalize the constitutive equations, we present the following similarity transformation:

$$
\begin{aligned}
& \hat{u}=\frac{a r}{2(1-a t)} f^{\prime}(\zeta), \quad \hat{w}=-\frac{a H}{\sqrt{1-a t}} f(\zeta), \quad \breve{N}=\frac{a r}{2 H(1-a t)^{3 / 2}} h(\zeta) \\
& B(t)=B_{0}(1-a t)^{-1 / 2}, \quad \zeta=\frac{z(1-a t)^{-1 / 2}}{H}, \quad \theta(\zeta)=\frac{\tilde{T}-\tilde{T}_{h}}{\tilde{T}_{w}-\tilde{T}_{h}}, \\
& \varphi(\zeta)=\frac{\tilde{C}-\tilde{C}_{h}}{\tilde{C}_{w}-\tilde{C}_{h}},
\end{aligned}
$$

Plugin (9) into Equs. (2)-(6), and eliminating the pressure gradient, we arrive at

$$
\begin{aligned}
& (1+K) f^{(i v)}-\frac{R}{2}\left(\xi f^{\prime \prime \prime}+3 f^{\prime \prime}-2 f f^{\prime \prime \prime}\right)-K h^{\prime \prime}-R M^{2} f^{\prime \prime}=0, \\
& \left(1+\frac{K}{2}\right) h^{\prime \prime}-R K\left(2 h-f^{\prime \prime}\right)-\frac{R}{2}\left(3 h+\zeta h^{\prime}+f^{\prime} h-2 f h^{\prime}\right)=0, \\
& \theta^{\prime \prime}+\operatorname{Pr} R\left(2 f \theta^{\prime}-\zeta \theta^{\prime}\right)+\operatorname{Pr} N b \theta^{\prime} \varphi^{\prime}+\operatorname{Pr} N t \theta^{\prime 2}=0, \\
& \varphi^{\prime \prime}+\operatorname{Le} R\left(2 f \varphi^{\prime}-\zeta \varphi^{\prime}\right)+\frac{N t}{N b} \theta^{\prime \prime}=0,
\end{aligned}
$$

with the boundary conditions

$$
\begin{aligned}
& f(0)=S, f^{\prime}(0)=0, h(0)=-n f^{\prime \prime}(0), \theta(0)=1, \\
& N b \theta^{\prime}(0)+N t \varphi^{\prime}(0)=0, f(1)=\frac{1}{2}, f^{\prime}(1)=0, \\
& h(1)=-n f^{\prime \prime}(1), \theta(1)=0, \varphi(1)=0 .
\end{aligned}
$$

Here $S$ denotes the suction/blowing parameter, squeeze Reynolds number $R$, Hartman number $M$, micropolar parameter $K$, Lewis number $L e$, Brownian motion coefficient $\mathrm{Nb}$, thermophoresis parameter $\mathrm{Nt}$, and Prandtl number $\mathrm{Pr}$ is defined as

$$
\begin{aligned}
& S=\frac{w_{0} \sqrt{1-a t}}{a H}, R=\frac{a H^{2}}{2 v}, M^{2}=\frac{\sigma B_{0}^{2}}{\rho a}, K=\frac{k}{\mu}, L e=\frac{v}{D_{B}}, \\
& N b=\frac{(\rho c)_{p} D_{B}\left(\tilde{C}_{w}-\tilde{C}_{h}\right)}{(\rho c)_{f} v}, N t=\frac{(\rho c)_{p} D_{T}\left(\tilde{T}_{w}-\tilde{T}_{h}\right)}{(\rho c)_{f} T_{m} v}, \operatorname{Pr}=\frac{v}{\alpha} .
\end{aligned}
$$

The skin friction coefficient, wall couple stress coefficient, local Nusselt and Sherwood numbers are defined as

$$
\begin{aligned}
& C_{f}=\frac{\tau_{r z}}{\rho\left(\frac{-a r}{1-a t}\right)^{2}}, C_{h}=\frac{H \gamma \frac{\partial \bar{N}}{\partial z}}{\rho\left(\frac{-a r}{1-a t}\right)^{2}}, N u=\frac{H q_{w}}{K\left(\tilde{T}_{w}-\tilde{T}_{h}\right)}, \\
& S h=\frac{H j_{w}}{D_{B}\left(\tilde{C}_{w}-\tilde{C}_{h}\right)},
\end{aligned}
$$

where

$$
\tau_{r z}=\frac{(\mu+k)}{\rho(a r)^{2}}\left(\frac{\partial \hat{u}}{\partial z}+\frac{\partial \hat{w}}{\partial r}\right), q_{w}=-k\left(\frac{\partial \tilde{T}}{\partial z}\right), j_{w}=-D_{B}\left(\frac{\partial \tilde{C}}{\partial z}\right) .
$$

With the help of (9) we get

$$
\begin{aligned}
& C_{1 f}=\frac{(1+K)}{R} f^{\prime \prime}(1), \quad C_{0 f}=\frac{(1+K)}{R} f^{\prime \prime}(0), \\
& C_{1 h}=-\frac{\left(1+\frac{K}{2}\right)}{R} h^{\prime}(1), C_{0 h}=-\frac{\left(1+\frac{K}{2}\right)}{R} h^{\prime}(1), \\
& N u r_{1}=N u_{1}(1-a t)^{1 / 2}=-\theta^{\prime}(1), \quad N u r_{0}=N u_{0}(1-a t)^{1 / 2}=-\theta^{\prime}(0), \\
& S h r_{1}=S h_{1}(1-a t)^{1 / 2}=-\varphi^{\prime}(1), \quad S h r_{0}=S h_{0}(1-a t)^{1 / 2}=-\varphi^{\prime}(0),
\end{aligned}
$$

\section{Solution Procedure for HAM}

For this problem, we use the HAM package BVPh2.0 
and considers the methodology proposed by Liao [31-33]. The initial guesses are

$$
\begin{aligned}
& f_{0}(\zeta)=S+\frac{3}{2}(1-2 S) \zeta^{2}+(2 S-1) \zeta^{3}, \\
& h_{0}(\zeta)=-3 n(-1+2 S)(2 \zeta-1), \\
& \theta_{0}(\zeta)=1-\zeta, \\
& \varphi_{0}(\zeta)=\frac{\mathrm{Nt}}{\mathrm{Nb}}(\zeta-1) .
\end{aligned}
$$

Linear operators are chosen as

$$
\ell_{f}=\frac{d^{4} f}{d \zeta^{4}}, \ell_{h}=\frac{d^{2} h}{d \zeta^{2}}, \ell_{\theta}=\frac{d^{2} \theta}{d \zeta^{2}} \text { and } \ell_{\phi}=\frac{d^{2} \phi}{d \zeta^{2}} .
$$

Above operators satisfies the following linearity conditions,

$$
\begin{aligned}
& \ell_{f}\left(\hat{A}_{1} \zeta^{3}+\hat{A}_{2} \zeta^{2}+\hat{A}_{3} \zeta+\hat{A}_{4}\right)=0, \\
& \ell_{h}\left(\hat{A}_{5} \zeta+\hat{A}_{6}\right)=0, \\
& \ell_{\theta}\left(\hat{A}_{7} \zeta+\hat{A}_{8}\right)=0, \\
& \ell_{\varphi}\left(\hat{A}_{9} \zeta+\hat{A}_{10}\right)=0,
\end{aligned}
$$

where $\hat{A}_{i}(i=1-10)$ are constants.

\section{Optimal Values of Convergence-control Parameters and Error Analysis}

Convergence criterion for the series solutions is highly

\begin{tabular}{|c|c|c|c|c|c|c|c|c|c|c|}
\hline$R$ & $M$ & $K$ & $N b$ & $N t$ & $L e$ & $h_{f}$ & $h_{h}$ & $h_{\theta}$ & $h_{\phi}$ & $\begin{array}{c}\text { Total Error } \\
\text { of the System }\end{array}$ \\
\hline 0.1 & 1.0 & 0.5 & 0.1 & 0.1 & 0.1 & -0.733 & -0.725 & -0.682 & -1.403 & $7.85 \times 10^{-5}$ \\
\hline 0.3 & & & & & & -0.710 & -0.710 & -0.699 & -1.421 & $4.44 \times 10^{-3}$ \\
\hline 0.5 & & & & & & -0.712 & -0.684 & -0.716 & -1.461 & $5.55 \times 10^{-2}$ \\
\hline 0.7 & & & & & & -0.725 & -0.659 & -0.724 & -1.524 & $3.50 \times 10^{-1}$ \\
\hline \multirow[t]{20}{*}{0.1} & 0.0 & & & & & -0.738 & -0.721 & -0.682 & -1.403 & $7.85 \times 10^{-5}$ \\
\hline & 1.0 & & & & & -0.733 & -0.725 & -0.682 & -1.403 & $7.85 \times 10^{-5}$ \\
\hline & 2.0 & & & & & -0.728 & -0.730 & -0.682 & -1.403 & $7.85 \times 10^{-5}$ \\
\hline & 3.0 & & & & & -0.724 & -0.734 & -0.682 & -1.403 & $7.85 \times 10^{-5}$ \\
\hline & 1.0 & 0.5 & & & & -0.733 & -0.725 & -0.682 & -1.403 & $7.85 \times 10^{-5}$ \\
\hline & & 1.0 & & & & -0.541 & -0.613 & -0.682 & -1.403 & $7.86 \times 10^{-5}$ \\
\hline & & 1.5 & & & & -0.425 & -0.532 & -0.682 & -1.403 & $7.86 \times 10^{-5}$ \\
\hline & & 2.0 & & & & -0.349 & -0.469 & -0.682 & -1.403 & $7.86 \times 10^{-5}$ \\
\hline & & 0.5 & 0.05 & & & -0.732 & -0.822 & -0.671 & -1.408 & $1.52 \times 10^{-4}$ \\
\hline & & & 0.1 & & & -0.733 & -0.725 & -0.682 & -1.403 & $7.85 \times 10^{-5}$ \\
\hline & & & 0.15 & & & -0.724 & -0.729 & -0.687 & -1.400 & $2.65 \times 10^{-5}$ \\
\hline & & & 0.2 & & & -0.711 & -0.735 & -0.689 & -1.396 & $5.86 \times 10^{-5}$ \\
\hline & & & 0.1 & 0.05 & & -0.740 & -0.716 & -1.390 & -0.613 & $1.13 \times 10^{-4}$ \\
\hline & & & & 0.1 & & -0.733 & -0.725 & -0.682 & -1.403 & $7.85 \times 10^{-5}$ \\
\hline & & & & 0.15 & & -0.735 & -0.815 & -0.611 & -1.488 & $3.48 \times 10^{-4}$ \\
\hline & & & & 0.2 & & -0.745 & -0.824 & -0.550 & -1.550 & $1.06 \times 10^{-3}$ \\
\hline & & & & 0.1 & 0.1 & -0.733 & -0.725 & -0.682 & -1.403 & $7.85 \times 10^{-5}$ \\
\hline & & & & & 0.4 & -0.730 & -0.727 & -0.684 & -1.400 & $6.98 \times 10^{-5}$ \\
\hline & & & & & 0.7 & -0.726 & -0.728 & -0.687 & -1.395 & $6.30 \times 10^{-5}$ \\
\hline & & & & & 1.0 & -0.719 & -0.732 & -0.689 & -1.391 & $5.77 \times 10^{-5}$ \\
\hline
\end{tabular}
dependent on the involved parameters. Here, we define total error of the system for $\widetilde{f}, \theta$ and $\phi$ to obtained the optimal values of such physical parameters as

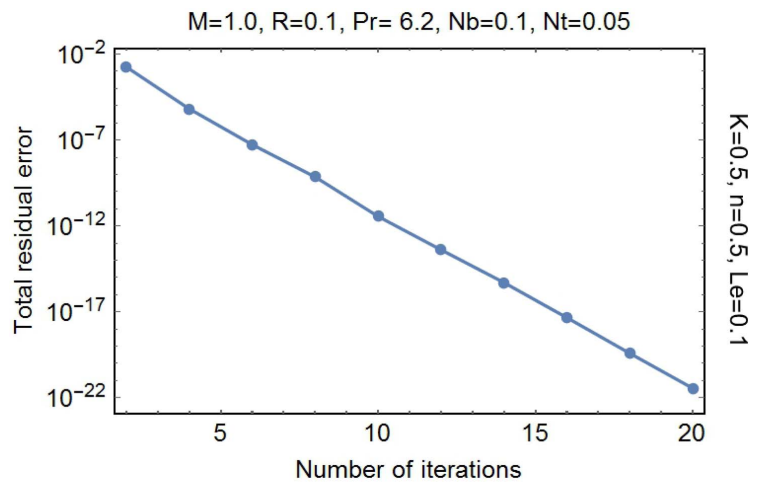

Fig. 2. (Color online) Total Residual Error vs. order of approximations.

Table 1. Optimal values of $h_{f}, h_{h}, h_{\theta}, h_{\phi}$ for different values of the physical parameters with $\operatorname{Pr}=6.2, n=0.5$ for suction case $(S>0)$. 
Table 2. Optimal values of $\hbar_{f}, \hbar_{h}, \hbar_{\theta}, \hbar_{\phi}$ for different values of the physical parameters with $\operatorname{Pr}=6.2, n=0.5$ for injection case $(S<0)$.

\begin{tabular}{|c|c|c|c|c|c|c|c|c|c|c|}
\hline$R$ & $M$ & $K$ & $\mathrm{Nb}$ & $N t$ & Le & $\hbar_{f}$ & $\hbar_{h}$ & $\hbar_{\theta}$ & $\hbar_{\phi}$ & $\begin{array}{l}\text { Total Error of } \\
\text { the System }\end{array}$ \\
\hline 0.1 & 1.0 & 0.5 & 0.1 & 0.1 & 0.1 & -0.616 & -0.785 & -0.661 & -1.407 & $7.85 \times 10^{-5}$ \\
\hline 0.3 & & & & & & -0.596 & -0.783 & -0.604 & -1.433 & $1.17 \times 10^{-3}$ \\
\hline 0.5 & & & & & & -0.584 & -0.794 & -0.561 & -1.460 & $3.58 \times 10^{-3}$ \\
\hline 0.7 & & & & & & -0.586 & -0.792 & -0.526 & -1.498 & $8.21 \times 10^{-3}$ \\
\hline \multirow[t]{20}{*}{0.1} & 0.0 & & & & & -0.621 & -0.780 & -0.661 & -1.407 & $7.85 \times 10^{-5}$ \\
\hline & 1.0 & & & & & -0.616 & -0.785 & -0.661 & -1.407 & $7.85 \times 10^{-5}$ \\
\hline & 2.0 & & & & & -0.605 & -0.799 & -0.661 & -1.407 & $7.85 \times 10^{-5}$ \\
\hline & 3.0 & & & & & -0.553 & -0.797 & -0.661 & -1.407 & $7.84 \times 10^{-5}$ \\
\hline & 1.0 & 0.5 & & & & -0.616 & -0.785 & -0.661 & -1.407 & $7.85 \times 10^{-5}$ \\
\hline & & 1.0 & & & & -0.470 & -0.665 & -0.661 & -1.407 & $7.84 \times 10^{-5}$ \\
\hline & & 1.5 & & & & -0.378 & -0.580 & -0.661 & -1.407 & $7.84 \times 10^{-5}$ \\
\hline & & 2.0 & & & & -0.315 & -0.511 & -0.661 & -1.407 & $7.84 \times 10^{-5}$ \\
\hline & & 0.5 & 0.05 & & & -0.610 & -0.774 & -0.620 & -1.407 & $2.35 \times 10^{-4}$ \\
\hline & & & 0.1 & & & -0.616 & -0.785 & -0.661 & -1.407 & $7.85 \times 10^{-5}$ \\
\hline & & & 0.15 & & & -0.535 & -0.885 & -1.081 & -0.447 & $2.80 \times 10^{-3}$ \\
\hline & & & 0.2 & & & -0.541 & -0.884 & -1.889 & -0.421 & $2.07 \times 10^{-3}$ \\
\hline & & & 0.1 & 0.05 & & -0.628 & -0.794 & -0.745 & -1.302 & $6.23 \times 10^{-6}$ \\
\hline & & & & 0.1 & & -0.616 & -0.785 & -0.661 & -1.407 & $7.85 \times 10^{-5}$ \\
\hline & & & & 0.15 & & -0.597 & -0.800 & -0.588 & -1.484 & $3.63 \times 10^{-4}$ \\
\hline & & & & 0.2 & & -0.585 & -0.804 & -0.499 & -1.528 & $9.50 \times 10^{-4}$ \\
\hline & & & & 0.1 & 0.1 & -0.616 & -0.785 & -0.661 & -1.407 & $7.85 \times 10^{-5}$ \\
\hline & & & & & 0.4 & -0.618 & -0.784 & -0.661 & -1.413 & $7.86 \times 10^{-5}$ \\
\hline & & & & & 0.7 & -0.619 & -0.783 & -0.661 & -1.419 & $7.84 \times 10^{-5}$ \\
\hline & & & & & 1.0 & -0.621 & -0.781 & -0.662 & -1.426 & $7.76 \times 10^{-5}$ \\
\hline
\end{tabular}

$$
\begin{aligned}
& E_{m, 1}\left(\hbar_{f}\right)=\frac{1}{K} \sum_{i=0}^{K}\left[N_{f}\left(\sum_{j=0}^{m} f_{j}(i \widehat{\Delta} x) \cdot \sum_{j=0}^{m} h_{j}(i \widehat{\Delta} x)\right)\right]^{2} \\
& E_{m, 2}\left(\hbar_{h}\right)=\frac{1}{K} \sum_{i=0}^{K}\left[N_{h}\left(\sum_{j=0}^{m} f_{j}(i \widehat{\Delta} x) \cdot \sum_{j=0}^{m} h_{j}(i \widehat{\Delta} x)\right)\right]^{2}, \\
& E_{m, 3}\left(\hbar_{\theta}\right)=\frac{1}{K} \sum_{i=0}^{K}\left[N_{\theta}\left(\sum_{j=0}^{m} f_{j}(i \widehat{\Delta} x) \cdot \sum_{j=0}^{m} \theta_{j}(i \widehat{\Delta} x) \cdot \sum_{j=0}^{m} \varphi_{j}(i \widehat{\Delta} x)\right)\right]^{2} \\
& E_{m, 4}\left(\hbar_{\varphi}\right)=\frac{1}{K} \sum_{i=0}^{K}\left[N_{\varphi}\left(\sum_{j=0}^{m} f_{j}(i \widehat{\Delta} x) \cdot \sum_{j=0}^{m} \theta_{j}(i \widehat{\Delta} x) \cdot \sum_{j=0}^{m} \varphi_{j}(i \widehat{\Delta} x)\right)\right]^{2}
\end{aligned}
$$

The highly nonlinear coupled system of differential equations (10)-(13) are solved using MATHEMATICA package BVPh2.0. In the so called BVPh2.0 the minimum squared residual errors at $20^{\text {th }}$ order of approximations are calculated as $5.26384971643049 \times 10^{-50}, 9.1635594296378$ $\times 10^{-53}, 1.8946211416852 \times 10^{-23}, 3.3901144623442 \times 10^{-22}$ for $f(\zeta), h(\zeta), \theta(\zeta)$ and $\varphi(\zeta)$ respectively. Figure 2 is sketched for the total residual error corresponding to the different order of approximations.

The optimal values of these auxiliary parameters for velocity, temperature and concentration profiles corresponding to the different values of the parameters are given in Tables 1 and 2 .

\section{Results and Discussion}

To highlight the influence of different physical parameters on the dimensionless velocities, temperature distribution and concentration profile are portrayed in Figs. 3-13. It is mentionable that $S>0$ corresponds to suction flow and while $S<0$ stands for blowing case. Effects of different emerging parameters such as micropolar parameter $K$, squeezed Reynolds number $R$ and Hartman number $M$ on radial velocity $f^{\prime}(\zeta)$ are displayed in Figs. $3-5$. Figure 3 depicts the influence of micropolar parameter $K$ on the radial velocity $f^{\prime}(\zeta)$. Here, one can easily see that in the case of suction $S>0$ radial velocity increases initially with increasing $K$, however when $\zeta$ approaches in the neighborhood of 0.4 , then it start decreasing with an increase in $K$. While, in the case of injection $S<0$ similar trend is observed for rising values of micropolar parameter $K$. Due to porosity of the lower disk when suction plays a prominent role, it allows the 


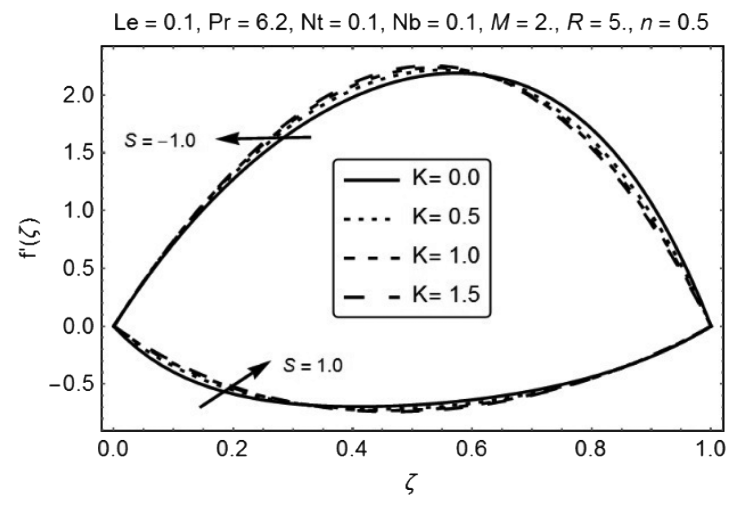

Fig. 3. Effect of $K$ on $f^{\prime}(\zeta)$.

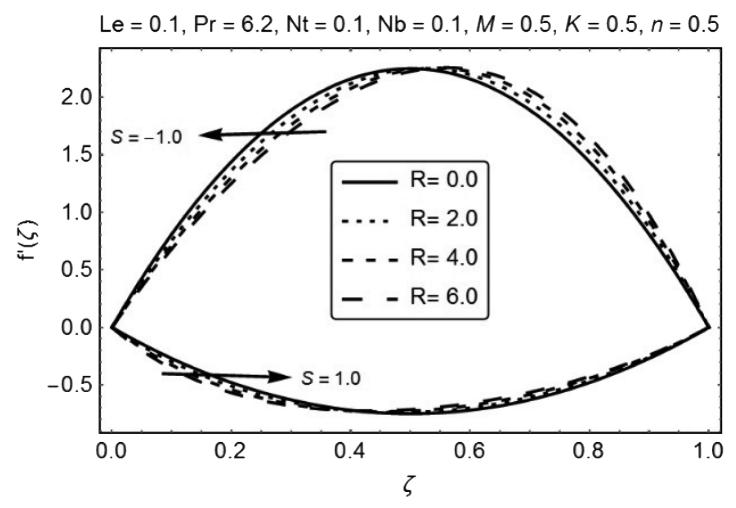

Fig. 4. Effect of $R$ on $f^{\prime}(\zeta)$.

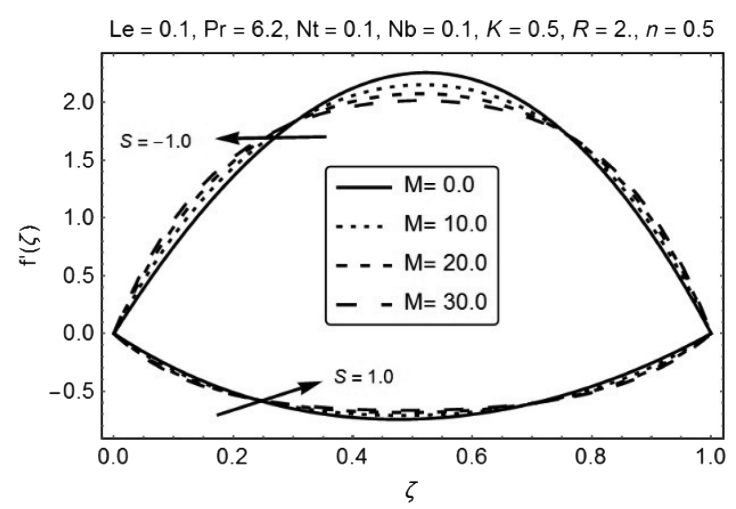

Fig. 5. Effect of $M$ on $f^{\prime}(\zeta)$.

fluid to flow near the walls which results in a thinner boundary layer. Influence of local squeezed Reynolds number $R$ on the radial velocity $f^{\prime}(\zeta)$ is portrayed in Fig. 4. It is clear from the Fig. 4 that the radial velocity $f^{\prime}(\zeta)$ increases for increasing values of $R$ in the range $0<\zeta \leq 0.5$, while an opposite trend is observed for $0.5<\zeta \leq 1$ for both the blowing $S<0$ and suction case $S$ $>0$.
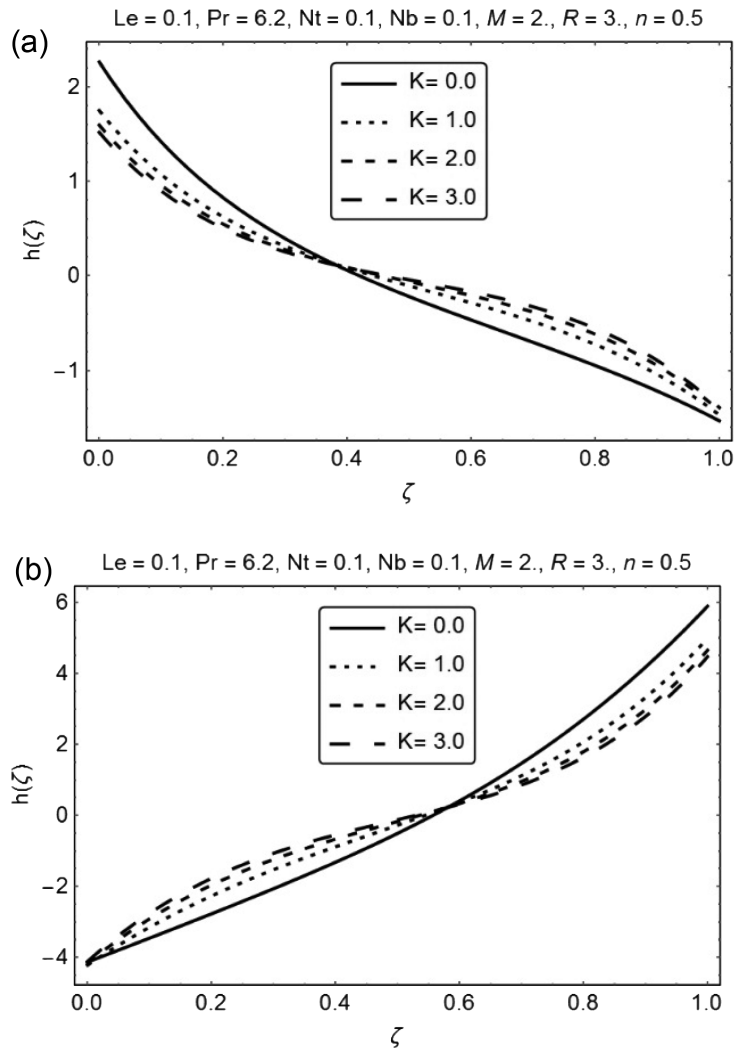

Fig. 6. (a) Effect of $K$ on $h(\zeta)$ for $S>0$. (b) Effect of $K$ on $h(\zeta)$ for $S<0$.

Figure 5 depicts the behavior of Hartmann number $M$ on the radial velocity $f^{\prime}(\zeta)$, it is observed that for the blowing case $S<0$ near the wall regions (i.e. $0<\zeta \leq 0.3$ and $0.8<\zeta \leq 1$ ) respectively, the velocity profile increase for increasing value of $M$, while, near the central region (i.e. $0.3<\zeta \leq 0.8$ ) the behavior of velocity profile is reversed. However, in the case of suction flow $S>0$, an opposite trend is observed with surging values of $M$.

To explore the effects of physical parameters on angular velocity $h(\zeta)$ (micro-motion) Figs. 6-7 are presented. Figure 6(a) reveals that in case of suction $S>0$, the angular velocity $h(\zeta)$ initially increases for increasing $K$, however when $\zeta$ approaches in the neighborhood of 0.4 , it starts decreasing for increasing values of $K$. While in Fig. 6 (b), the reverse behavior is shown for rising values of micropolar parameter $K$ in the case of blowing $S<0$. Influence of local squeezed Reynolds number $R$ on the angular velocity is depicted in Fig. 7. From the Fig. 7(a) it is clear that near the wall regions (i.e. $0<\zeta \leq 0.1$ and $0.65<\zeta \leq 1)$, the angular velocity $h(\zeta)$ increases with increasing value of $R$ whereas in the central region (i.e. $0.1<\zeta \leq 0.65)$, the angular velocity decreasing for increasing values of $R$ for the suction $S>0$. However, in Fig. 7(b) a similar trend is observed in the angular 
(a) $\operatorname{Le}=0.1, \operatorname{Pr}=6.2, \mathrm{Nt}=0.1, \mathrm{Nb}=0.1, M=2 ., K=0.5, n=0.5$

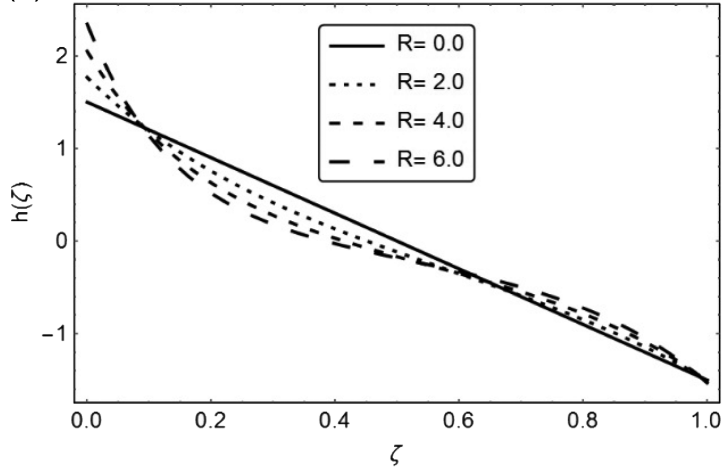

(b) Le $=0.1, \operatorname{Pr}=6.2, \mathrm{Nt}=0.1, \mathrm{Nb}=0.1, M=2 ., K=0.5, n=0.5$

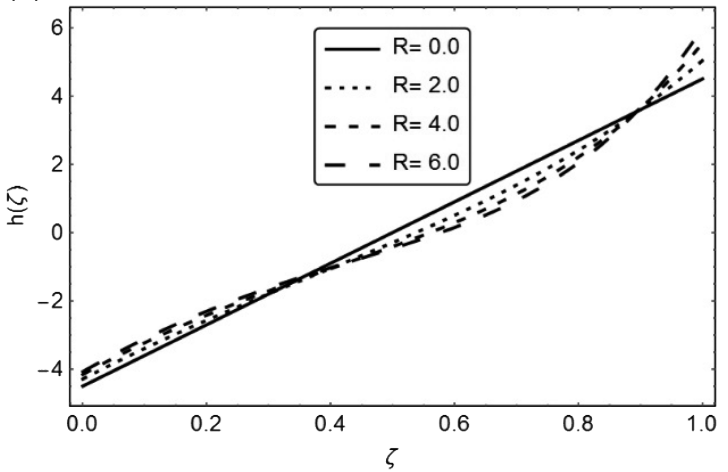

Fig. 7. (a) Effect of $R$ on $h(\zeta)$ for $S>0$. (b) Effect of $R$ on $h(\zeta)$ for $S<0$.

velocity for higher values of local squeezed Reynolds number in the case of blowing $S<0$.

Behavior of temperature distribution $\theta(\zeta)$ under the influence of pertinent flow parameters such as local squeezed Reynolds number $R$ and thermophoresis parameter $N t$ are discussed in Figs. 8-9. In Fig. 8, the temperature distribution $\theta(\zeta)$ is directly proportional to $R$ in the case of blowing $S<0$ whereas $\theta(\zeta)$ is inversely proportional to local squeezed Reynolds number $R$ for the suction case $S$ $>0$. While in Fig. 9, an opposite trend is observed in the

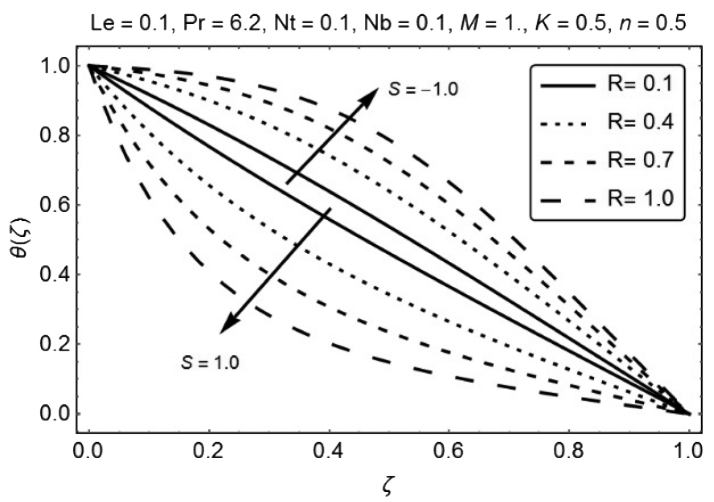

Fig. 8. Effect of $R$ on $\theta(\zeta)$.

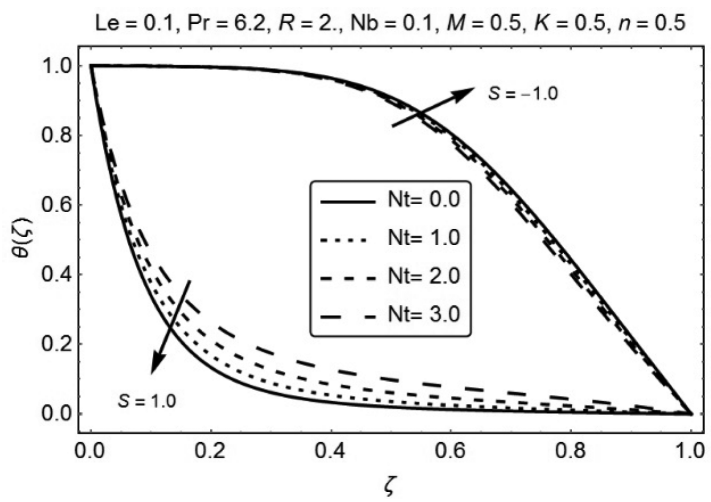

Fig. 9. Effect of $N t$ on $\theta(\zeta)$.

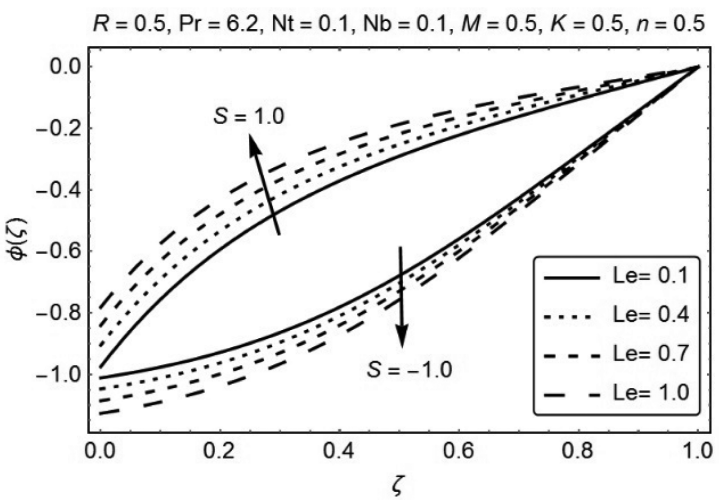

Fig. 10. Effect of $L e$ on $\phi(\zeta)$.

temperature distribution $\theta(\zeta)$ with rising values of thermophoresis parameter $N t$ for both the suction $S>0$ and blowing $S<0$ cases.

To examine the effects of different physical parameter on concentration profile $\phi(\zeta)$ are displayed in Figs. 1013. Figure 10 reveals that concentration profile $\phi(\zeta)$ is directly proportional to Lewis number $L e$ in the case of suction $S>0$, while in the case of injection $S<0$ variation in $\phi(\zeta)$ is inversely proportional to the Lewis

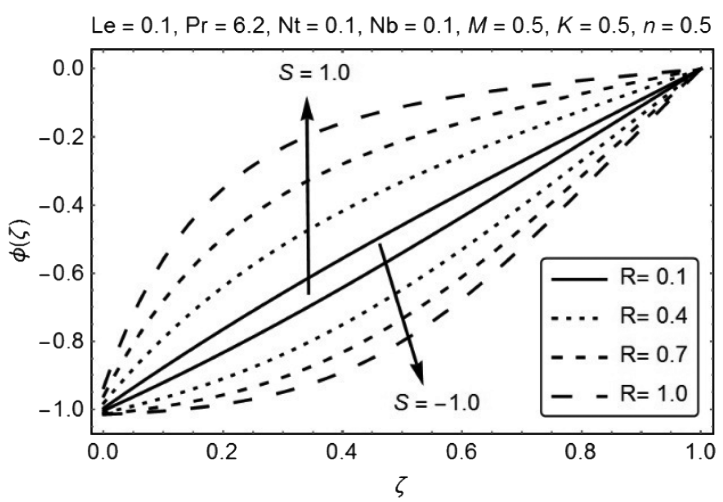

Fig. 11. Effect of $R$ on $\phi(\zeta)$. 


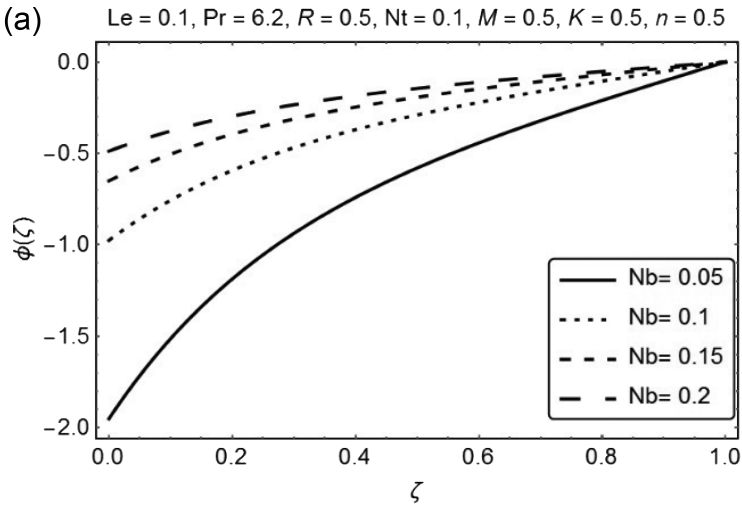

(b) $\quad \mathrm{Le}=0.1, \mathrm{Pr}=6.2, R=0.5, \mathrm{Nt}=0.1, M=0.5, K=0.5, n=0.5$

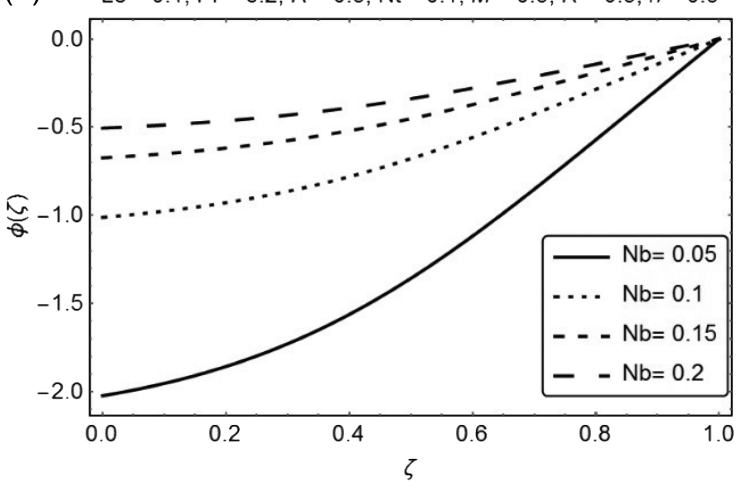

Fig. 12. (a) Effect of $N b$ on $\phi(\zeta)$ for $S>0$. (b) Effect of $N b$ on $\phi(\zeta)$ for $S<0$.

number Le. Figure 11 depicts the behavior of local squeezed Reynolds number $R$ on the concentration profile is opposite to that accounted for temperature profile $\theta(\zeta)$ in Fig. 8 for both the suction $S>0$ and injection $S$ $<0$ cases.

In Fig. 12, the effect of Brownian motion parameter on concentration profile $\phi(\zeta)$ is presented. It is observed that the concentration profile rapidly increases with increas-

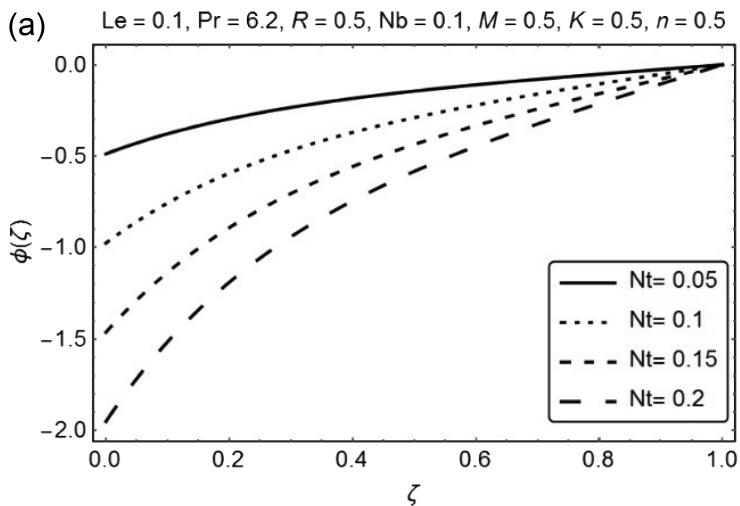

(b) Le $=0.1, \operatorname{Pr}=6.2, R=0.5, \mathrm{Nb}=0.1, M=0.5, K=0.5, n=0.5$

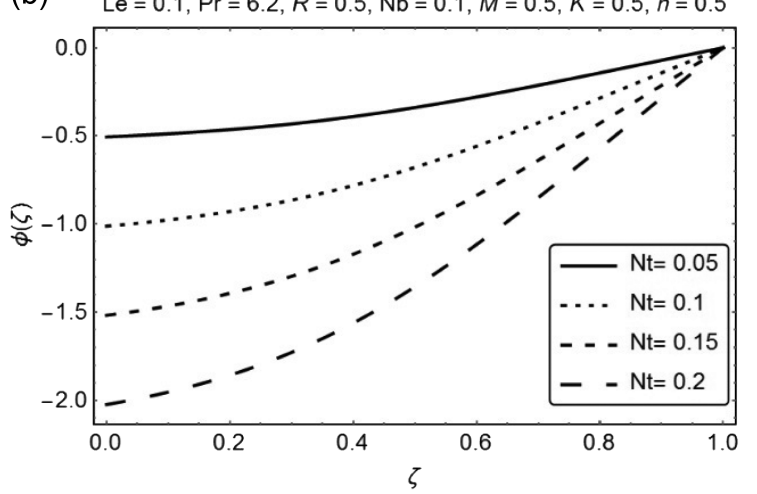

Fig. 13. (a) Effect of $N t$ on $\phi(\zeta)$ for $S>0$. (b) Effect of $N t$ on $\phi(\zeta)$ for $S<0$.

ing values of Brownian motion parameter $N b$ for both the suction $S>0$ and blowing $S<0$ cases. While, Fig. 13 reveals that the influence of thermophoresis parameter $N t$ on the concentration profile $\phi(\zeta)$ is opposite to that accounted for Brownian motion parameter $\mathrm{Nb}$ in both the suction $S>0$ and blowing $S<0$ cases.

Furthermore, to check the validity and efficiency of HAM we solve the similar problem with Runge-Kutta

Table 3. Comparison of HAM and Numerical Solution for dimensionless velocities $f(\zeta)$ and $h(\zeta)$.

\begin{tabular}{|c|c|c|c|c|c|c|}
\hline \multirow{2}{*}{$\zeta$} & \multicolumn{3}{|c|}{$f(\zeta)$} & \multicolumn{3}{|c|}{$h(\zeta)$} \\
\hline & HAM & RK4 & Absolute Error & HAM & RK4 & Absolute Error \\
\hline 0.0 & 1.000000 & 1.000000 & $1.116160 \times 10^{-8}$ & 1.553170 & 1.553170 & $1.055710 \times 10^{-7}$ \\
\hline 0.1 & 0.985601 & 0.985601 & $9.345960 \times 10^{-9}$ & 1.189840 & 1.189840 & $9.007810 \times 10^{-8}$ \\
\hline 0.2 & 0.946842 & 0.946842 & $9.064500 \times 10^{-9}$ & 0.855711 & 0.855710 & $7.608970 \times 10^{-8}$ \\
\hline 0.3 & 0.890180 & 0.890180 & $1.010820 \times 10^{-8}$ & 0.543844 & 0.543843 & $6.351270 \times 10^{-8}$ \\
\hline 0.4 & 0.821851 & 0.821851 & $1.221060 \times 10^{-8}$ & 0.248068 & 0.248068 & $5.206650 \times 10^{-8}$ \\
\hline 0.5 & 0.747914 & 0.747914 & $1.509270 \times 10^{-8}$ & -0.037230 & -0.037230 & $4.145860 \times 10^{-8}$ \\
\hline 0.6 & 0.674302 & 0.674302 & $1.850870 \times 10^{-8}$ & -0.317279 & -0.317279 & $3.115110 \times 10^{-8}$ \\
\hline 0.7 & 0.606870 & 0.606870 & $2.226050 \times 10^{-8}$ & -0.597102 & -0.597102 & $2.124090 \times 10^{-8}$ \\
\hline 0.8 & 0.551441 & 0.551441 & $2.624340 \times 10^{-8}$ & -0.881669 & -0.881669 & $1.169970 \times 10^{-8}$ \\
\hline 0.9 & 0.513854 & 0.513854 & $3.061030 \times 10^{-8}$ & -1.176050 & -1.176050 & $3.454570 \times 10^{-9}$ \\
\hline 1.0 & 0.500000 & 0.500000 & 0.0 & -1.485580 & -1.485580 & $3.635310 \times 10^{-8}$ \\
\hline
\end{tabular}


Table 4. Comparison of HAM and Numerical Solution for dimensionless temperature $\theta(\zeta)$ and concentration $\phi(\zeta)$.

\begin{tabular}{|c|c|c|c|c|c|c|}
\hline \multirow{2}{*}{$\zeta$} & \multicolumn{3}{|c|}{$\theta(\zeta)$} & \multicolumn{3}{|c|}{$\phi(\zeta)$} \\
\hline & HAM & RK4 & Absolute Error & HAM & RK4 & Absolute Error \\
\hline 0.0 & 1.000000 & 1.000000 & $4.567030 \times 10^{-9}$ & -0.975233 & -0.975233 & $3.775820 \times 10^{-7}$ \\
\hline 0.1 & 0.780338 & 0.780337 & $3.106400 \times 10^{-8}$ & -0.756136 & -0.756136 & $3.404190 \times 10^{-7}$ \\
\hline 0.2 & 0.615866 & 0.615865 & $8.165690 \times 10^{-8}$ & -0.593116 & -0.593116 & $2.527580 \times 10^{-7}$ \\
\hline 0.3 & 0.489255 & 0.489255 & $1.453490 \times 10^{-7}$ & -0.468564 & -0.468565 & $1.203880 \times 10^{-7}$ \\
\hline 0.4 & 0.388500 & 0.388499 & $1.849390 \times 10^{-7}$ & -0.370278 & -0.370278 & $7.478360 \times 10^{-9}$ \\
\hline 0.5 & 0.305312 & 0.305312 & $1.762260 \times 10^{-7}$ & -0.289838 & -0.289838 & $7.834590 \times 10^{-8}$ \\
\hline 0.6 & 0.233934 & 0.233933 & $1.266550 \times 10^{-7}$ & -0.221392 & -0.221392 & $7.957110 \times 10^{-8}$ \\
\hline 0.7 & 0.170289 & 0.170289 & $7.383430 \times 10^{-8}$ & -0.160802 & -0.160802 & $5.013870 \times 10^{-8}$ \\
\hline 0.8 & 0.111435 & 0.111435 & $3.715530 \times 10^{-8}$ & -0.105077 & -0.105077 & $2.236840 \times 10^{-8}$ \\
\hline 0.9 & 0.055203 & 0.055203 & $1.580880 \times 10^{-8}$ & -0.052015 & -0.052015 & $6.995140 \times 10^{-9}$ \\
\hline 1.0 & 0.0 & 0.0 & 0.0 & 0.0 & 0.0 & 0.0 \\
\hline
\end{tabular}

method of order four. The comparisons of developed algorithm and numerical solution are presented in Table 3 and 4. It is evident from the tables that an excellent agreement found between the analytical solution and numerical solution.

To investigate the effects of different emerging parameters on skin friction coefficient with strong and weak interactions (i.e. $n=0$ and $n=0.5$ ) at upper and lower disks are portrayed in Tables 5-6. It is clearly seen that for increasing values of Hartmann number $M$ an incline is observed in the skin friction coefficient at upper disk, while a decline is shown at lower disk with increasing values of $M$ in the case of suction $S>0$. However, in the case of blowing $S<0$, opposite trend is observed at the upper and lower disks for both the strong and weak interactions. For suction $S>0$ and blowing $S<0$ the skin friction coefficient at upper and lower disks decreases with surging values of local squeezed Reynolds number $R$ to both the strong and weak interactions. While, the effects of micropolar parameter $K$ on the skin friction coefficient at upper and lower disks respectively, is similar to that accounted for Hartmann number $M$. As a result, it is mentionable that skin friction coefficient at upper disk is higher than the lower disk with increasing values of $M$ and $K$ for the suction case $S>0$, while, in the blowing case $S<0$, this trend is observed opposite for surging values of Hartmann number $M$ and micropolar parameter $K$ in both the strong and weak interactions.

To explore the effects of different pertinent parameters on wall couple stress coefficients for both the strong and weak interactions at lower and upper disk are presented in Tables 7, 8. It is evident from the tabulated values that the wall couple stress coefficients in the presence of weak and strong interactions at upper disk are the increasing Table 5. Skin friction coefficient $\frac{(1+K)}{R} f^{\prime \prime}(1)$ and $\frac{(1+K)}{R} f^{\prime \prime}(0)$ at upper and lower disks for strong interaction $n=0.0$ with
$\operatorname{Pr}=6.2$.

\begin{tabular}{|c|c|c|c|c|c|c|c|c|c|}
\hline & & & & & & $\frac{(1+K)}{R} f^{\prime \prime}(1)$ & $\frac{(1+K)}{R} f^{\prime \prime}(0)$ & $\frac{(1+K)}{R} f^{\prime \prime}(1)$ & $\frac{(1+K)}{R} f^{\prime \prime}(0)$ \\
\hline$M$ & $R$ & $K$ & $\mathrm{Nb}$ & $N t$ & Le & \multicolumn{2}{|c|}{ for suction $(S>0)$} & \multicolumn{2}{|c|}{ for injection $(S<0)$} \\
\hline 0.0 & 1.0 & 0.5 & 0.1 & 0.1 & 0.1 & 4.37795564 & -4.88711811 & -14.22882334 & 12.78623413 \\
\hline 2.0 & & & & & & 4.48220137 & -4.98048135 & -14.50273182 & 13.08898140 \\
\hline 3.0 & & & & & & 4.53348558 & -5.02664200 & -14.638200740 & 13.23804300 \\
\hline 4.0 & & & & & & 4.58423077 & -5.07246058 & -14.77269449 & 13.38561616 \\
\hline 1.0 & 0.0 & & & & & 4.50000171 & -4.50000145 & -13.50000185 & 13.50000140 \\
\hline & 2.0 & & & & & 4.37698401 & -5.39000800 & -15.20174390 & 12.48076203 \\
\hline & 3.0 & & & & & 4.33909137 & -5.86566941 & -16.00563771 & 12.10944533 \\
\hline & 4.0 & & & & & 4.31548330 & -6.35857489 & -16.77856486 & 11.80826988 \\
\hline & 1.0 & 0.0 & & & & 2.94502918 & -3.44727992 & -9.88099045 & 8.49295168 \\
\hline & & 1.0 & & & & 5.90455664 & -6.41103470 & -18.81183494 & 17.35773888 \\
\hline & & 1.5 & & & & 7.37259318 & -7.88184872 & -23.23406190 & 21.76088210 \\
\hline & & 2.0 & & & & 8.83688311 & -9.34855799 & -27.64144405 & 26.15361197 \\
\hline
\end{tabular}


Table 6. Skin friction coefficient $\frac{(1+K)}{R} f^{\prime \prime}(1)$ and $\frac{(1+K)}{R} f^{\prime \prime}(0)$ at upper and lower disks for weak interaction $n=0.5$ with
$\operatorname{Pr}=6.2$

\begin{tabular}{|c|c|c|c|c|c|c|c|c|c|}
\hline & & & & & & $\frac{(1+K)}{R} f^{\prime \prime}(1)$ & $\overline{\frac{(1+K)}{R} f^{\prime \prime}(0)}$ & $\frac{(1+K)}{R} f^{\prime \prime}(1)$ & $\frac{(1+K)}{R} f^{\prime \prime}(0)$ \\
\hline$M$ & $R$ & $K$ & $\mathrm{Nb}$ & $N t$ & $\mathrm{Le}$ & \multicolumn{2}{|c|}{ for suction $(S>0)$} & \multicolumn{2}{|c|}{ for injection $(S<0)$} \\
\hline 0.0 & 1.0 & 0.5 & 0.1 & 0.1 & 0.1 & 4.38836492 & -4.80161596 & -14.04977009 & 12.85510598 \\
\hline 2.0 & & & & & & 4.49208087 & -4.89477185 & -14.32368995 & 13.15651312 \\
\hline 3.0 & & & & & & 4.54311245 & -4.94082833 & -14.45914636 & 13.30493609 \\
\hline 4.0 & & & & & & 4.59361266 & -4.98654263 & -14.59361679 & 13.45189019 \\
\hline 1.0 & 0.0 & & & & & 4.50000171 & -4.50000145 & -13.50000185 & 13.50000140 \\
\hline & 2.0 & & & & & 4.38839971 & -5.21660516 & -14.87419700 & 12.58005722 \\
\hline & 3.0 & & & & & 4.34560405 & -5.60289694 & -15.54930030 & 12.22111821 \\
\hline & 4.0 & & & & & 4.31286062 & -6.00540693 & -16.20798440 & 11.92223875 \\
\hline & 1.0 & 0.0 & & & & 2.94502918 & -3.44727992 & -9.88099044 & 8.49295168 \\
\hline & & 1.0 & & & & 5.90360013 & -6.25945435 & -18.47807283 & 17.42376771 \\
\hline & & 1.5 & & & & 7.34969624 & -7.67298143 & -22.75569648 & 21.78577978 \\
\hline & & 2.0 & & & & 8.78583287 & -9.08693637 & -27.02354315 & 26.11371143 \\
\hline
\end{tabular}
Table 7. Wall couple stress coefficient $-\frac{\left(1+\frac{K}{2}\right)}{R} h^{\prime}(1)$ and $-\frac{\left(1+\frac{K}{2}\right)}{R} h^{\prime}(0)$ at upper and lower disks for strong interaction $n=0.0$
with $\operatorname{Pr}=6.2$.

\begin{tabular}{|c|c|c|c|c|c|c|c|c|c|}
\hline & & & & & & $\frac{\left(1+\frac{K}{2}\right)}{R} h^{\prime}(1)$ & $-\frac{\left(1+\frac{K}{2}\right)}{R} h^{\prime}(0)$ & $-\frac{\left(1+\frac{K}{2}\right)}{R} h^{\prime}(1)$ & $-\frac{\left(1+\frac{K}{2}\right)}{R} h^{\prime}(0)$ \\
\hline$M$ & $R$ & $K$ & $\mathrm{Nb}$ & $N t$ & $\mathrm{Le}$ & \multicolumn{2}{|c|}{ for suction $(S>0)$} & \multicolumn{2}{|c|}{ for injection $(S<0)$} \\
\hline 0.0 & 1.0 & 0.5 & 0.1 & 0.1 & 0.1 & 0.24706349 & 0.24945778 & -0.70931906 & -0.68609316 \\
\hline 2.0 & & & & & & 0.24712127 & 0.24945890 & -0.70957381 & -0.68686255 \\
\hline 3.0 & & & & & & 0.24714887 & 0.24945954 & -0.70970081 & -0.68723545 \\
\hline 4.0 & & & & & & 0.24717567 & 0.24946021 & -0.70982749 & -0.68760085 \\
\hline 1.0 & 0.5 & & & & & 0.12452383 & 0.12484361 & -0.36346120 & -0.36001685 \\
\hline & 1.0 & & & & & 0.24709282 & 0.24945832 & -0.70944653 & -0.68648190 \\
\hline & 1.5 & & & & & 0.36656806 & 0.37395800 & -1.04360778 & -0.97798676 \\
\hline & 2.0 & & & & & 0.48220741 & 0.49844019 & -1.36936641 & -1.23599139 \\
\hline & 1.0 & 0.5 & & & & 0.24709282 & 0.24945832 & -0.70944653 & -0.68648190 \\
\hline & & 1.0 & & & & 0.48945673 & 0.49511935 & -1.41728189 & -1.38004618 \\
\hline & & 1.5 & & & & 0.72935901 & 0.73802695 & -2.12411214 & -2.07701856 \\
\hline & & 2.0 & & & & 0.96781130 & 0.97903550 & -2.83026642 & -2.77591747 \\
\hline
\end{tabular}

functions of Hartmann number $M$, local squeezed Reynolds number $R$ and micropolar parameter $K$ in case of suction $S>0$ and decreasing functions at lower disk. However, the reverse phenomena is occurred in case of blowing $S<$ 0 From the table one can easily see that wall couple stress coefficient at upper disk is higher than the lower disk for all the emerging flow parameters in case of suction $S>0$, while, an opposite behavior is shown in the case of blowing $S<0$ for both the strong and weak interactions.

To investigate the effects of local Nusselt number under the influence of physical parameters with strong and weak interactions at upper and lower disks are constructed in
Tables 9, 10. For increasing values of local squeezed Reynolds number $R$ the rate of heat transfer decreases at upper disk in the presence of strong and weak interactions for the suction $S>0$ and increases with an increase in $R$ at lower disk. While in the blowing $S<0$ variations in local Nusselt number are opposite at upper and lower disk. For rising values of Brownian motion parameter $\mathrm{Nb}$ the rate of heat transfer is decreasing function for both the suction $S>0$ and blowing $S<0$ cases at lower and upper disk respectively. However, the local Nusselt number is directly proportional to thermophoresis parameter $N t$ only in the case of suction $S>0$ at upper disk. 

Table 8. Wall couple stress coefficient $-\frac{\left(1+\frac{K}{2}\right)}{R} h^{\prime}(1)$ and $-\frac{\left(1+\frac{K}{2}\right)}{R} h^{\prime}(0)$ at upper and lower disks for weak interaction $n=0.5$
with $\operatorname{Pr}=6.2$.

\begin{tabular}{|c|c|c|c|c|c|c|c|c|c|}
\hline & & & & & & $\frac{\left(1+\frac{K}{2}\right)}{R} h^{\prime}(1)$ & $\frac{\left(1+\frac{K}{2}\right)}{R} h^{\prime}(0)$ & $-\frac{\left(1+\frac{K}{2}\right)}{R} h^{\prime}(1)$ & $-\frac{\left(1+\frac{K}{2}\right)}{R} h^{\prime}(0)$ \\
\hline$M$ & $R$ & $K$ & $N b$ & $N t$ & Le & \multicolumn{2}{|c|}{ for suction $(S>0)$} & \multicolumn{2}{|c|}{ for injection $(S<0)$} \\
\hline 0.0 & 1.0 & 0.5 & 0.1 & 0.1 & 0.1 & 4.21510914 & 5.78953126 & -15.19087787 & -11.16126856 \\
\hline 2.0 & & & & & & 4.30276857 & 5.90429822 & -15.49200534 & -11.40077400 \\
\hline 3.0 & & & & & & 4.34595475 & 5.96093214 & -15.64077394 & -11.51886840 \\
\hline 4.0 & & & & & & 4.38872534 & 6.01707882 & -15.78837252 & -11.63589062 \\
\hline 1.0 & 0.5 & & & & & 3.99758428 & 4.76815590 & -13.34015944 & -11.22134051 \\
\hline & 1.0 & & & & & 4.25915683 & 5.84716776 & -15.34204675 & -11.28158260 \\
\hline & 1.5 & & & & & 4.53007047 & 6.98976386 & -17.28904606 & -11.38462812 \\
\hline & 2.0 & & & & & 4.80683420 & 8.19846326 & -19.19997703 & -11.50635489 \\
\hline & 1.0 & 0.5 & & & & 4.25915683 & 5.84716776 & -15.34204675 & -11.28158260 \\
\hline & & 1.0 & & & & 5.45032179 & 6.95616408 & -18.78767801 & -14.82637153 \\
\hline & & 1.5 & & & & 6.63338738 & 8.08473001 & -22.24739004 & -18.35083250 \\
\hline & & 2.0 & & & & 7.81080893 & 9.22356806 & -25.71408294 & -21.86334863 \\
\hline
\end{tabular}

Table 9. Local Nusselt number $-\theta^{\prime}(1)$ and $-\theta^{\prime}(0)$ at upper and lower disks for strong interaction $n=0.0$ with $\operatorname{Pr}=6.2$.

\begin{tabular}{|c|c|c|c|c|c|c|c|c|c|}
\hline \multirow[b]{2}{*}{$R$} & \multirow[b]{2}{*}{$\mathrm{Nb}$} & \multirow[b]{2}{*}{$N t$} & \multirow[b]{2}{*}{ Le } & \multirow[b]{2}{*}{$M$} & \multirow[b]{2}{*}{$K$} & $-\theta^{\prime}(1)$ & $-\theta^{\prime}(0)$ & $-\theta^{\prime}(1)$ & $-\theta^{\prime}(0)$ \\
\hline & & & & & & \multicolumn{2}{|c|}{ for suction $(S>0)$} & \multicolumn{2}{|c|}{ for injection $(S<0)$} \\
\hline 0.5 & 0.1 & 0.1 & 0.1 & 1.0 & 0.5 & 0.55119983 & 2.54658646 & 1.40758330 & 0.29712181 \\
\hline 1.0 & & & & & & 0.24078399 & 5.05206995 & 1.73847898 & 0.07526472 \\
\hline 1.5 & & & & & & 0.08631338 & 8.12261856 & 1.99750715 & 0.01731961 \\
\hline 2.0 & & & & & & 0.02708852 & 11.34438013 & 2.20691837 & 0.00375335 \\
\hline 1.0 & 0.05 & & & & & 0.24078401 & 5.05206787 & 1.73847899 & 0.07526473 \\
\hline & 0.1 & & & & & 0.24078399 & 5.05206995 & 1.73847898 & 0.07526472 \\
\hline & 0.15 & & & & & 0.24078398 & 5.05207038 & 1.73847898 & 0.07526472 \\
\hline & 0.2 & & & & & 0.24078398 & 5.05207077 & 1.73847898 & 0.07526473 \\
\hline & 0.1 & 0.05 & & & & 0.23688410 & 5.06956706 & 1.74270220 & 0.07509303 \\
\hline & & 0.1 & & & & 0.24078399 & 5.05206995 & 1.73847898 & 0.07526472 \\
\hline & & 0.15 & & & & 0.24472697 & 5.03461129 & 1.73425004 & 0.07543730 \\
\hline & & 0.2 & & & & 0.24871305 & 5.01718987 & 1.73001535 & 0.07561070 \\
\hline
\end{tabular}

Table 10. Local Nusselt number $-\theta^{\prime}(1)$ and $-\theta^{\prime}(0)$ at upper and lower disks for weak interaction $n=0.5$ with $\operatorname{Pr}=6.2$.

\begin{tabular}{|c|c|c|c|c|c|c|c|c|c|}
\hline & & & & & & $-\theta^{\prime}(1)$ & $-\theta^{\prime}(0)$ & $-\theta^{\prime}(1)$ & $-\theta^{\prime}(0)$ \\
\hline$R$ & $\mathrm{Nb}$ & $N t$ & Le & $M$ & $K$ & \multicolumn{2}{|c|}{ for suction $(S>0)$} & \multicolumn{2}{|c|}{ for injection $(S<0)$} \\
\hline 0.5 & 0.1 & 0.1 & 0.1 & 1.0 & 0.5 & 0.55092459 & 2.54734931 & 1.40632820 & 0.29753959 \\
\hline 1.0 & & & & & & 0.24022099 & 5.05534730 & 1.73417794 & 0.07570481 \\
\hline 1.5 & & & & & & 0.08583207 & 8.12845052 & 1.98930458 & 0.01754496 \\
\hline 2.0 & & & & & & 0.02682165 & 11.35148445 & 2.19440217 & 0.00383728 \\
\hline 1.0 & 0.05 & & & & & 0.24022101 & 5.05534377 & 1.73417794 & 0.07570481 \\
\hline & 0.1 & & & & & 0.24022099 & 5.05534730 & 1.73417794 & 0.07570481 \\
\hline & 0.15 & & & & & 0.24022098 & 5.05534773 & 1.73417793 & 0.07570481 \\
\hline & 0.2 & & & & & 0.24022098 & 5.05534814 & 1.73417793 & 0.07570481 \\
\hline & 0.1 & 0.05 & & & & 0.23632615 & 5.07284718 & 1.73838906 & 0.07553222 \\
\hline & & 0.1 & & & & 0.24022099 & 5.05534730 & 1.73417794 & 0.07570481 \\
\hline & & 0.15 & & & & 0.24415896 & 5.03788570 & 1.72996110 & 0.07587827 \\
\hline & & 0.2 & & & & 0.24814006 & 5.02046119 & 1.72573852 & 0.07605261 \\
\hline
\end{tabular}


Table 11. Local Sherwood number $-\phi^{\prime}(1)$ and $-\phi^{\prime}(0)$ at upper and lower disks for strong interaction $n=0.0$ with $\operatorname{Pr}=6.2$.

\begin{tabular}{|c|c|c|c|c|c|c|c|c|c|}
\hline \multirow{3}{*}{$\frac{R}{0.5}$} & \multirow{3}{*}{$\frac{N b}{0.1}$} & \multirow{3}{*}{$\begin{array}{l}N t \\
0.1\end{array}$} & \multirow{3}{*}{$\begin{array}{l}\mathrm{Le} \\
0.1\end{array}$} & \multirow{3}{*}{$\begin{array}{c}M \\
1.0\end{array}$} & \multirow{3}{*}{$\frac{K}{0.5}$} & $-\phi^{\prime}(1)$ & $-\phi^{\prime}(0)$ & $-\phi^{\prime}(1)$ & $-\phi^{\prime}(0)$ \\
\hline & & & & & & \multicolumn{2}{|c|}{ for suction $(S>0)$} & \multicolumn{2}{|c|}{ for injection $(S<0)$} \\
\hline & & & & & & -0.51931167 & -2.54658646 & -1.42580510 & -0.29712181 \\
\hline 1.0 & & & & & & -0.16548454 & -5.05207004 & -1.76590150 & -0.07526472 \\
\hline 1.5 & & & & & & 0.03656064 & -8.12261883 & -2.03021496 & -0.01731961 \\
\hline 2.0 & & & & & & 0.14181764 & -11.34438105 & -2.24333110 & -0.00375335 \\
\hline \multirow[t]{12}{*}{1.0} & 0.05 & & & & & -0.33096912 & -10.10413584 & -3.531803001 & -0.15052947 \\
\hline & 0.1 & & & & & -0.16548454 & -5.05207004 & -1.76590150 & -0.07526472 \\
\hline & 0.15 & & & & & -0.11032302 & -3.36804698 & -1.17726766 & -0.05017648 \\
\hline & 0.2 & & & & & -0.08274227 & -2.52603544 & -0.88295075 & -0.03763236 \\
\hline & 0.1 & 0.05 & & & & -0.08073484 & -2.53478358 & -0.88504314 & -0.03754652 \\
\hline & & 0.1 & & & & -0.16548454 & -5.05207004 & -1.76590150 & -0.07526472 \\
\hline & & 0.15 & & & & -0.25431435 & -7.55191705 & -2.64256669 & -0.11315595 \\
\hline & & 0.2 & & & & -0.34728945 & -10.03437983 & -3.51503031 & -0.15122141 \\
\hline & & 0.1 & 0.1 & & & -0.16548454 & -5.05207004 & -1.76590150 & -0.07526472 \\
\hline & & & 0.4 & & & 0.00612877 & -4.95471651 & -1.82706632 & -0.07636970 \\
\hline & & & 0.7 & & & 0.13880183 & -4.86801568 & -1.89539044 & -0.07760608 \\
\hline & & & 1.0 & & & 0.24055586 & -4.79078296 & -1.97214369 & -0.07899738 \\
\hline
\end{tabular}

Table 12. Local Sherwood number $-\phi^{\prime}(1)$ and $-\phi^{\prime}(0)$ at upper and lower disks for weak interaction $n=0.5$ with $\operatorname{Pr}=6.2$.

\begin{tabular}{|c|c|c|c|c|c|c|c|c|c|}
\hline & & & & & & $-\phi^{\prime}(1)$ & $-\phi^{\prime}(0)$ & $-\phi^{\prime}(1)$ & $-\phi^{\prime}(0)$ \\
\hline$R$ & $N b$ & $N t$ & Le & $M$ & $K$ & \multicolumn{2}{|c|}{ for suction $(S>0)$} & \multicolumn{2}{|c|}{ for injection $(S<0)$} \\
\hline 0.5 & 0.1 & 0.1 & 0.1 & 1.0 & 0.5 & -0.51902018 & -2.54734931 & -1.42452244 & -0.29753959 \\
\hline 1.0 & & & & & & -0.16486488 & -5.05534738 & -1.76152207 & -0.07570481 \\
\hline 1.5 & & & & & & 0.03712603 & -8.12845079 & -2.02187320 & -0.01754496 \\
\hline 2.0 & & & & & & 0.14216551 & -11.35148552 & -2.23060697 & -0.00383728 \\
\hline 1.0 & 0.05 & & & & & -0.32972980 & -10.11068760 & -3.52304416 & -0.15140962 \\
\hline & 0.1 & & & & & -0.16486488 & -5.05534738 & -1.76152207 & -0.07570481 \\
\hline & 0.15 & & & & & -0.10990992 & -3.37023188 & -1.17434805 & -0.05046987 \\
\hline & 0.2 & & & & & -0.08243243 & -2.52767412 & -0.88076103 & -0.03785241 \\
\hline & 0.1 & 0.05 & & & & -0.08042760 & -2.53642364 & -0.88284740 & -0.03776611 \\
\hline & & 0.1 & & & & -0.16486488 & -5.05534738 & -1.76152207 & -0.07570481 \\
\hline & & 0.15 & & & & -0.25337715 & -7.55682866 & -2.63601564 & -0.11381741 \\
\hline & & 0.2 & & & & -0.34602965 & -10.04092247 & -3.50631971 & -0.15210522 \\
\hline & & 0.1 & 0.1 & & & -0.16486488 & -5.05534738 & -1.76152207 & -0.07570481 \\
\hline & & & 0.4 & & & 0.00689264 & -4.95797802 & -1.82250996 & -0.07681553 \\
\hline & & & 0.7 & & & 0.13964339 & -4.87126233 & -1.89063177 & -0.07805820 \\
\hline & & & 1.0 & & & 0.24142681 & -4.79401605 & -1.96715134 & -0.07945642 \\
\hline
\end{tabular}

To validate the effects of pertinent flow parameters on local Sherwood number at upper and lower disks in the presence of weak and strong interactions Tables 11, 12 are displayed. Here, one can easily see that the rate of mass transfer at upper disk increases with increasing values of local squeezed Reynolds number $R$ in the case of suction $S>0$ whereas a fall is observed at the lower disk for both the strong and weak interactions. However, an opposite behavior is shown in the case of blowing $S<$ 0 . Local Sherwood number is directly proportional to
Brownian motion parameter $\mathrm{Nb}$ for both the suction $S>0$ and blowing $S<0$ in the presence of strong and weak interactions at lower and upper disks respectively. For increasing values of thermophoresis parameter $\mathrm{Nt}$, the rate of mass transfer is increasing function for suction flow $S$ $>0$ at upper disk to both the weak and strong interactions and vice versa. However, the local Sherwood number is directly proportional to Lewis number $L e$ for suction $S>$ 0 and blowing $S<0$ at upper and lower disks in both the weak and strong interactions. 


\section{Conclusions}

In the current study, squeezing flow of micropolar nanofluid between parallel disks is analyzed. HAM package BVPh2.0 has been employed to solve the highly nonlinear ordinary differential equations that governs the flow and control the convergence of series solution. Strong and weak interactions (i.e. $n=0$ and $n=0.5$ ) is taken into account for the numerical values of skin friction coefficient, wall couple stress coefficient, local Nusselt number and local Sherwood number at upper and lower disks respectively. To check the validity and reliability of developed algorithm (HAM Package BVPh2.0) we seek a numerical solution using Runge-Kutta method order of four which shows an excellent agreement between these analytical and numerical solution. Graphical aids of different physical parameters on dimensionless velocities, temperature distribution and concentration profile is also a part of this study. The main features of the current study are captured as follows;

- Radial velocity $f^{\prime}(\zeta)$ is directly proportional to the variations of micropolar parameter $K$ for both the suction $S$ $>0$ and blowing $S<0$ when $\zeta$ approaches in the neighborhood of 0.4 , and inversely proportional to $K$ in the region $0.4<\zeta \leq 1$.

- Effect of local squeezed Reynolds number $R$ on the velocity profile is inversely proportional to that accounted for micropolar parameter $K$ in the case of suction $S>0$ and blowing $S>0$.

- Angular velocity $h(\zeta)$ increases for increasing value of micropolar parameter $K$ when $\zeta$ reaches the neighborhood of 0.55 and it starts decreasing for rising values of $K$ in the blowing case $S<0$, while the opposite trend is observed in the case of suction $S>0$.

- Temperature profile $\theta(\zeta)$ is directly proportional to thermophoresis parameter $N t$ in the case of suction $S>$ 0 , and inversely proportional to $N t$ for blowing $S<0$ case.

- An increase in Lewis number Le increases the concentration profile $\theta(\zeta)$ in the suction case $S>0$, while in the blowing flow $S<0$ concentration profile decreases for increasing values of $L e$.

- In the presence of weak and strong interactions (i.e. $n=0$ and $n=0.5$ ) for both the suction $S>0$ and blowing $S<0$ cases, numerical values of skin friction coefficient, wall coupe stress coefficient, local Nusselt number and Sherwood number are presented in tabulated form.

\section{References}

[1] K. R. Rajagopal, Acta Sin. Indica 18, 1 (1982).
[2] K. R. Rajagopal, Navier-Stokes Equation and Related Nonlinear Problems, A. Sequira, ed., Plenum Press, New York (1995) pp. 273-278.

[3] K. R. Rajagopal, A. Z. Szeri, and W. Troy, Int. J. NonLinear Mech. 21, 279 (1986).

[4] C. Fetecau, C. Fetecau, and D. Vieru, Acta Mech. 189, 53 (2007).

[5] T. Hayat, Z. Abbas, and N. Ali, Phys. Lett. A 372, 4698 (2008).

[6] M. Ayub, A. Rasheed, and T. Hayat, Int. J. Eng. Sci. 41, 2091 (2003).

[7] T. Hayat, R. Ellahi, and S. Asghar, Chem. Commun. 193, 1 (2008).

[8] M. Sajid, T. Hayat, S. Asghar, and K. Vajravelu, Arch. Appl. Mech. 78, 127 (2008).

[9] A. C. Eringen, J. Math. 16, 1 (1966).

[10] T. Ariman, M. A. Turk, and N. D. Sylvester, Int. J. Eng. Sci. 12, 273 (1974).

[11] M. A. Ezzat, M. I. Othman, and K. A. Helmy, Can. J. Phys. 77, 813 (1999).

[12] K. A. Helmy, H. F. Idriss, and S. E. Kassem, Can. J. Phys. 80, 166 (2002).

[13] D. A. S. Rees and I. Pop, IMA. J. Appl. Math. 61, 179 (1998).

[14] S. K. Jena and M. N. Mathur, Int. J. Eng. 19, 1431 (1981).

[15] T. Hayat, Z. Abbas, and T. Javed, Phys. Lett. A 372, 637 (2008).

[16] G. S. Guram and A. C. Smith, Comput. Math. Appl. 6, 213 (1980).

[17] G. Ahmadi, Int. J. Eng. Sci. 14, 639 (1976).

[18] R. Nazar, N. Amin, D. Filip, and I. Pop, Int. J. Non-Linear Mech. 39, 1227 (2004).

[19] H. S. Takhar, R. Bhargava, R. S. Agrawal, and A. V. S. Balaji, Int. J. Eng. Sci. 38, 1907 (2000).

[20] S. Ishizawa, Bull. Jpn. Soc. Mech. Eng. 9, 533 (1966).

[21] R. J. Grimm, App. Sci. Res. 32, 149 (1976).

[22] C. Y. Wang and L. T. Watson, App. Sci. Res. 35, 195 (1979).

[23] R. Usha and R. Sridharan, Fluid Dyn. Res. 18, 35 (1999).

[24] M. M. Rashidi, H. Shahmohamadi, and S. Dinarvand, Math. Probl. Eng. 2008, 935095 (2008).

[25] S. U. S. Choi, Siginer, D. A. Wang, H. P. (Eds.), Developments and Applications of Non-Newtonian Flows, 231, 99 (1995).

[26] J. Buongiorno, J. Heat Transfer 128, 240 (2006).

[27] M. Sheikholeslami and R. Ellahi, Applied Sciences 5, 294 (2015).

[28] M. Sheikholeslami and R. Ellahi, International Journal of Heat and Mass Transfer 89, 799 (2015).

[29] S. T. Mohyud-Din, Z. A. Zaidi, U. Khan, and N. Ahmed, Aerospace Science and Technology 46, 514 (2015).

[30] N. Ahmed, S. T. Mohyud-Din, and S. M. Hassan, Aerospace Science and Technology 48, 53 (2016).

[31] S. J. Liao, Beyond Perturbation: Introduction to Homo- 
topy Analysis Method, Chapman and Hall, CRC Press, Boca Raton.

[32] S. J. Liao, Applied Mathematics and Computation 147, 499 (2004).

[33] S. J. Liao, Commun. Nonlinear Sci. Numer. Simul. 15,
2003 (2010).

[34] U. Khan, N. Ahmed, and S. T. Mohyud-Din, Chemical Engineering Science 141, 17 (2016).

[35] S. T. Mohyud-Din and S. I. U. Khan, Aerospace Science and Technology 48, 186 (2016). 